8-1-2007

\title{
Matrix analysis of classical elastic collisions
}

John H. Carter

Missouri State University

Michael Lieber

Follow this and additional works at: https://bearworks.missouristate.edu/articles-cnas

\section{Recommended Citation}

Carter, John H., and Michael Lieber. "Matrix analysis of classical elastic collisions." American Journal of Physics 75, no. 8 (2007): 758-759.

This article or document was made available through BearWorks, the institutional repository of Missouri State University. The work contained in it may be protected by copyright and require permission of the copyright holder for reuse or redistribution.

For more information, please contact BearWorks@library.missouristate.edu. 


\title{
Matrix analysis of classical elastic collisions
}

\author{
John H. Carter ${ }^{\mathrm{a})}$ and Michael Lieber \\ Department of Physics, University of Arkansas, Fayetteville, Arkansas 72701
}

(Received 29 January 2003; accepted 27 April 2007)

The velocities of two bodies before and after an elastic collision can be related by a matrix transformation in one and two dimensions. We demonstrate a very simple relation between the oneand two-dimensional collision matrices. (c) 2007 American Association of Physics Teachers.

[DOI: $10.1119 / 1.2742395]$

\section{INTRODUCTION}

The standard texts in classical mechanics ${ }^{1-3}$ treat binary collisions in the center of mass frame and then discuss scattering by a central force without offering a method for dealing with collisions in arbitrary inertial frames. This note presents a technique for handling such cases in two dimensions using complex scattering matrices. The technique has several applications in the classroom including how complex numbers can be useful in classical mechanics, a field where the use of complex numbers is rare. The method also gives a classical context to certain ideas that are more commonly associated with quantum mechanics, such as state vectors and operators, thus allowing these topics to be introduced earlier in the curriculum. Finally, the method can be used in mathematical physics classes to provide an illustration of matrix exponentiation with a real power, in which the matrix and exponent each have a clear physical meaning.

When a particle of mass $m$ and velocity $u$ and a particle of mass $M$ and velocity $v$ collide elastically in one dimension, their velocities after the collision, $u^{\prime}$ and $v^{\prime}$, respectively, are uniquely determined by $u$ and $v$. The reason is that $u^{\prime}$ and $v^{\prime}$ are determined from the conservation equations for energy and linear momentum. These equations are

$$
\begin{aligned}
& m u+M v=m u^{\prime}+M v^{\prime}, \\
& \frac{1}{2} m u^{2}+\frac{1}{2} M v^{2}=\frac{1}{2} m u^{\prime 2}+\frac{1}{2} M v^{\prime 2},
\end{aligned}
$$

and are readily solved. If we ignore the trivial nonscattering solution, $u^{\prime}=u$ and $v^{\prime}=v$, the final velocities may be expressed in terms of the initial ones and the appropriate scattering matrix $S_{1}$ as

$$
\left(\begin{array}{l}
u^{\prime} \\
v^{\prime}
\end{array}\right)=S_{1}\left(\begin{array}{l}
u \\
v
\end{array}\right)=\frac{1}{m+M}\left(\begin{array}{cc}
m-M & 2 M \\
2 m & M-m
\end{array}\right)\left(\begin{array}{l}
u \\
v
\end{array}\right) .
$$

In two dimensions, where there are four unknown velocity components, two for each particle, but only three conservation equations (one for energy and two for linear momentum), there is one remaining degree of freedom in the solution. This degree of freedom may be interpreted as the scattering angle, $\alpha$, of the collision in the center of mass frame. The natural scattering matrix would be a $4 \times 4$ matrix function of $\alpha$. However, if we represent the particle velocities by complex numbers, we can find a $2 \times 2$ complex matrix, $S_{2}$, which transforms the precollision velocities to the postcollision velocities. We now further analyze the one- dimensional case and demonstrate the relation between $S_{1}$ and $S_{2}$. The eigenvalues of $S_{1}$ are 1 and -1 with normalized eigenvectors:

$$
\begin{aligned}
& \mathbf{e}_{+}=\frac{1}{\sqrt{2}}\left(\begin{array}{l}
1 \\
1
\end{array}\right), \\
& \mathbf{e}_{-}=\frac{1}{\sqrt{m^{2}+M^{2}}}\left(\begin{array}{c}
M \\
-m
\end{array}\right),
\end{aligned}
$$

respectively. The matrix can be written in standard spectrally decomposed form as

$$
S_{1}=(+1) P_{+1}+(-1) P_{-1},
$$

where

$$
\begin{aligned}
& P_{+1}=\frac{1}{(m+M)}\left(\begin{array}{ll}
m & M \\
m & M
\end{array}\right), \\
& P_{-1}=\frac{1}{(m+M)}\left(\begin{array}{cc}
M & -M \\
-m & m
\end{array}\right)
\end{aligned}
$$

are the projection operators onto the eigenvectors $\mathbf{e}_{+}$and $\mathbf{e}_{-}$, respectively. The coefficient of -1 in Eq. (5) serves to flip the velocities in the center of mass frame.

Equation (5) may be interpreted as follows. The projector $P_{+1}$ extracts the velocity of the center of mass frame, that is,

$$
P_{+1}\left(\begin{array}{l}
u \\
v
\end{array}\right)=\left(\begin{array}{l}
w_{\mathrm{cm}} \\
w_{\mathrm{cm}}
\end{array}\right)
$$

where $w_{\mathrm{cm}}$ is the velocity of the center of mass frame. Because $w_{\mathrm{cm}}$ is unchanged in the collision, this projector has a coefficient of +1 . The projector $P_{-1}$ extracts the velocities as they appear in the center of mass frame, that is,

$$
P_{-1}\left(\begin{array}{l}
u \\
v
\end{array}\right)=\left(\begin{array}{l}
u-w_{\mathrm{cm}} \\
v-w_{\mathrm{cm}}
\end{array}\right) \text {. }
$$

In the center of mass frame, the effect of the collision is to flip the signs of the velocities; this is handled by the coefficient of -1 in front of $P_{-1}$.

We now consider the two-dimensional case and modify Eq. (5) to obtain the equation for $S_{2}$. In two dimensions, as in one, the total momentum of the particles in the center of mass frame is zero, before and after the collision. This fact, together with conservation of energy, implies that the collision may only rotate the velocities through an angle, which is 
the scattering angle. If the velocities before the collision in the center of mass frame are $u$ and $v$, where $v=-(m / M) u$, then after the collision they are $u \exp (i \alpha)$ and $v \exp (i \alpha)$. Thus, to obtain $S_{2}$, we only have to replace the -1 of Eq. (5) by $\exp (i \alpha)$. We also may obtain $S_{2}$ by exponentiating $S_{1}$ as we now show. Because they are projection operators, $P_{+1}$ and $P_{-1}$ are idempotent matrices, that is, they are equal to their square. Also, their product with one another is the zero matrix: $P_{+1} P_{-1}=P_{-1} P_{+1}=0$. It follows that their linear combination, $S_{1}$, may be raised to an arbitrary power by raising the eigenvalues 1 and -1 to that same power. The replacement $-1 \rightarrow \exp (i \alpha)$ is accomplished if $S_{1}$ is raised to the power $\alpha / \pi$, because $(-1)^{\alpha / \pi}=\exp (i \alpha)$ :

$$
S_{2}=S_{1}^{(\alpha / \pi)}=\frac{1}{m+M}\left(\begin{array}{cc}
m+M r & M(1-r) \\
m(1-r) & M+m r
\end{array}\right),
$$

which depends only on $m, M$, and $\alpha$ with $r=\exp (i \alpha)$. Although this matrix was derived by reasoning in the center of mass frame, it works equally well in any frame of reference-the $P_{+1}$ projector handles the transformation into and out of the center of mass frame. Two special cases of Eq. (9) are evident. If $\alpha=0$, then no collision occurs, and $S_{2}$ is the identity matrix. If $\alpha=\pi$, then $S_{2}=S_{1}$. That is, the collision occurs and is confined to one dimension. Two-dimensional collisions in which the particles' velocities change direction are intermediate between these special cases.
Note that the one- and two-dimensional scattering matrices, $S_{1}$ and $S_{2}$, respectively, depend only on the mass ratios of the particles and not on their velocities. Thus the final velocities are linear functions of the initial velocities. In three dimensions we cannot write down a matrix that rotates a vector through an angle without knowing the axis of rotation. Hence, in three dimensions the final velocities are not linear functions of the initial velocities, and so the sort of linear matrix operation described in this note cannot be applied.

\section{ACKNOWLEDGMENT}

The results in this paper were found in the course of doctoral research performed at the University of Arkansas with the support of a graduate research fellowship from the University of Arkansas.

\footnotetext{
${ }^{a}$ Present address: Department of Physics, Astronomy and Materials Science, Missouri State University, Springfield, MO 65897.

${ }^{1}$ H. Goldstein, Classical Mechanics (Addison-Wesley, Reading, 1980), pp. 105-119, 148-158.

${ }^{2}$ L. D. Landau and E. M. Lifshitz, Mechanics (Pergamon, Oxford, 1960), pp. 44-57.

${ }^{3}$ K. Symon, Mechanics (Addison-Wesley, Reading, MA, 1971), pp. 175188.
}

\title{
Comment on "An entangled web of crime: Bell's theorem as a short story," by K. Jacobs and H. M. Wiseman [Am. J. Phys. 73 (10), 932-937 (2005)]
}

\author{
P. K. Aravind ${ }^{a)}$ \\ Department of Physics, Worcester Polytechnic Institute, Worcester, Massachusetts 01609
}

(Received 6 February 2007; accepted 6 April 2007)

[DOI: 10.1119/1.2735632]

I enjoyed the exploits of Mr. Doyle and Dr. Bell in the paper by Jacobs and Wiseman. ${ }^{1}$ However, there is a shorter solution to Problem 1 than given in Ref. 1. Referring to Table 1 of Ref. 1 and keeping the testimony of the guards in mind, we see that there is an odd number of reds in each of the first three rows and an even number in the last row. Thus, the total number of reds in the table, when summed by the rows, is odd+odd+odd+even, which is odd. If we look at the columns, we see that both sides of each robber appear twice in the column under him, implying that the total number of reds in each column is even. Thus, if we sum by the columns, the total number of reds in the table is even+even+even, which is even. The contradiction with the earlier conclusion implies that one of the guards must either be mistaken or lying.

The fact that the statements of any three guards are always consistent can be seen as follows. Consider only the rows of the table associated with these guards and note that either the back or front of a robber occurs twice in each column of this truncated table. If these "doubled" entries are highlighted, it will be seen that there is exactly one unhighlighted entry in each row. Thus, no matter what choices of colors are made for the highlighted (or doubled) entries, it is clear that the unhighlighted entries can always be chosen so as to make the total number of reds in each row either even or odd, as dictated by that particular guard's testimony. A visual inspection of the table shows that this situation holds no matter which subset of three guards is selected. This argument also shows that there are exactly eight ways in which the robbers can be attired if the statements of a particular set of three guards are to be consistent with each other (the eight ways correspond to the $2^{3}$ choices of colors for the three doubled entries).

The statements of any two guards can be made consistent with each other by suitably coloring the backs and fronts of two robbers, but only one side of the third; this coloring can be done in eight ways. Adding a third guard requires us to color the previously unexposed side of the third robber, but the number of possibilities still remains at eight. However, adding on the fourth guard causes the number of possibilities to shrink to zero.

\footnotetext{
${ }^{a)}$ Electronic mail: paravind@wpi.edu

${ }^{1} \mathrm{~K}$. Jacobs and H. M. Wiseman, "An entangled web of crime: Bell's theorem as a short story,” Am. J. Phys. 73, 932-937 (2005).
} 